\title{
High-throughput discovery of high-temperature conventional superconductors
}

\author{
Alice M. Shipley $\odot,{ }^{*}, \dagger$ Michael J. Hutcheon $\odot,,, \ddagger$ and Richard J. Needs \\ Theory of Condensed Matter Group, Cavendish Laboratory, J. J. Thomson Avenue, Cambridge CB3 OHE, United Kingdom \\ Chris J. Pickard ${ }^{\S}$ \\ Department of Materials Science and Metallurgy, 27 Charles Babbage Rd., Cambridge CB3 OFS, United Kingdom \\ and Advanced Institute for Materials Research, Tohoku University, 2-1-1 Katahira, Aoba, Sendai 980-8577, Japan
}

(Received 9 April 2021; revised 21 May 2021; accepted 22 July 2021; published 4 August 2021)

\begin{abstract}
We survey the landscape of binary hydrides across the entire periodic table from 10 to $500 \mathrm{GPa}$ using a crystal structure prediction method. Building a critical temperature $\left(T_{c}\right)$ model, with inputs arising from density of states calculations and Gaspari-Gyorffy theory, allows us to predict which energetically competitive candidates are most promising for high- $T_{c}$ superconductivity. Implementing optimizations, which lead to an order of magnitude speedup for electron-phonon calculations, then allows us to perform an unprecedented number of "high-throughput" calculations of $T_{c}$ based on these predictions and to refine the model in an iterative manner. Converged electron-phonon calculations are performed for 121 of the best candidates from the final model. From these, we identify 36 above-100 K dynamically stable superconductors. To the best of our knowledge, superconductivity has not been previously studied in 27 of these. Of the 36,18 exhibit superconductivity above $200 \mathrm{~K}$, including structures of $\mathrm{NaH}_{6}(248-279 \mathrm{~K})$ and $\mathrm{CaH}_{6}(216-253 \mathrm{~K})$ at the relatively low pressure of $100 \mathrm{GPa}$.
\end{abstract}

DOI: 10.1103/PhysRevB.104.054501

\section{INTRODUCTION}

A number of dense metal hydrides have been shown to be conventional superconductors with high critical temperatures $\left(T_{c}\right)$ [1-6]. However, electron-phonon calculations, used to determine the $T_{c}$ of such materials from first principles, remain computationally expensive. It is not necessarily clear before performing these calculations which particular systems and structures might exhibit high-temperature superconductivity. Coupled with this, a huge variety of hydride stoichiometries and structures are either stable or metastable under pressure, even when the discussion is limited solely to binaries. This means that exhaustive theoretical investigation of this class of materials is a huge challenge. A combined searching and screening protocol could therefore provide a desirable and efficient way to discover new high- $T_{c}$ superconductors.

In this work, we use a crystal structure prediction method to explore binary hydrides of elements from across the entire periodic table over a 10-500 GPa pressure range. Stable and metastable structures are screened using a model built to predict $T_{c}$ from inputs including electronic density of states (DOS) ratios and electron-phonon coupling estimates from Gaspari-Gyorffy theory [7]. High-throughput electronphonon calculations are then performed for a large number of structures, selected based on the predictions of this model.

\footnotetext{
*These authors contributed equally to this work.

†ams277@cam.ac.uk

${ }^{\ddagger}$ mjh261@cam.ac.uk

§cjp20@cam.ac.uk
}

This provides more $T_{c}$ results which can be fed back into the training set, allowing us to improve the model iteratively. The results of this high-throughput model-training stage allow us to identify the most promising candidate systems at each pressure; more thorough structure searching is then carried out for each of these systems and fully converged electron-phonon calculations are performed for the best predicted candidates. A large number of high- $T_{c}$ superconductors are efficiently identified, including many not reported in previous work.

\section{METHODOLOGY}

\section{A. Initial structure searching}

The structure searching calculations in this work were performed using $a b$ initio random structure searching (AIRSS) $[8,9]$ and the plane-wave pseudopotential code CASTEP [10]. The Perdew-Burke-Ernzerhof generalized gradient approximation [11], CASTEP QC5 pseudopotentials, a 340-eV plane-wave cutoff, and a k-point spacing of $2 \pi \times 0.07 \AA^{-1}$ were used. For the initial searches, sp-AIRSS [12] was utilized and structures with 8-48 symmetry operations were generated. This served the dual purpose of (1) reducing the computational cost of the searches and subsequent calculations during the training phase and (2) putting additional focus on high-symmetry structures (which may be metastable or stabilized at nonzero temperatures). Throughout this work, we aim to consider the broadest set of compositions possible. To this end, we construct a convex hull for binary hydrides of the form $X_{n} H_{m}$ for every element $X$ in the periodic table at 10,100,200,300, and $500 \mathrm{GPa}$ in order to assess their 
stability. Structures on or near these static-lattice convex hulls were then selected for further investigation at the relevant pressure. In particular, in the training phase, stoichiometries within $80 \mathrm{meV} /$ f.u. of the hull were selected (for each of these stoichiometries just the lowest energy structure was chosen). In order to discuss stability more clearly in this work, we introduce two quantities: $E_{\text {stoic }}$, the distance of the given stoichiometry from the static-lattice convex hull, and $E_{\text {struc }}$, the distance of the given structure from the lowest energy structure of the same stoichiometry. Here, for example, we are selecting structures with $E_{\text {stoic }} \leqslant 80 \mathrm{meV} /$ f.u. and $E_{\text {struc }}=0$.

\section{B. Electron-phonon coupling estimates}

McMillan [13] showed that for strong-coupled superconductors the electron-phonon coupling constant, $\lambda$, can be expressed as

$$
\lambda=\frac{N\left(E_{F}\right)\left\langle I^{2}\right\rangle}{M\left\langle\omega^{2}\right\rangle},
$$

where $N\left(E_{F}\right)$ is the DOS at the Fermi level, $\left\langle I^{2}\right\rangle$ is the Fermi surface averaged electron-phonon matrix element, $M$ is the atomic mass, and $\left\langle\omega^{2}\right\rangle$ is the average squared phonon frequency. The numerator in this expression can be relabeled as $\eta$, the so-called Hopfield parameter. In situations where the vibrational modes can be well separated into those of different atomic character (as we typically see in binary hydrides) we can write

$$
\lambda=\sum_{j} \lambda_{j}=\sum_{j} \frac{\eta_{j}}{M_{j}\left\langle\omega_{j}^{2}\right\rangle},
$$

where $j$ is the atom type.

Hopfield established that, in a local phonon representation, electron-phonon interactions mainly consist of scatterings that change the electronic angular momentum $l$ [14]; building on this observation, Gaspari-Gyorffy theory [7] then provides a way to approximate $\left\langle I^{2}\right\rangle$ in Eq. (1). Beginning from a multiple-scattering Green's function formalism, adopting the rigid muffin-tin approximation [15] allows a number of approximations concerning the potential and wave function to be applied [16]. These approximations reduce the expression for the Hopfield parameter to a combination of electronic scattering phase shifts and (partial) electronic densities of states [16]. Recent work has emerged using Gaspari-Gyorffy theory for metal hydrides under high pressure [16,17].

The final form of the rigid-ion Gaspari-Gyorffy formula is

$$
\begin{aligned}
\left\langle I^{2}\right\rangle= & \frac{E_{F}}{\pi^{2} N^{2}\left(E_{F}\right)} \\
& \times \sum_{l} \frac{2(l+1) \sin ^{2}\left(\delta_{l+1}-\delta_{l}\right) N_{l}\left(E_{F}\right) N_{l+1}\left(E_{F}\right)}{N_{l}^{(1)} N_{l+1}^{(1)}},
\end{aligned}
$$

where $N_{l}^{(1)}$ is the free-scatterer DOS-the DOS at the Fermi level for a single muffin-tin potential in a zero-potential background-and $\delta_{l}$ are the scattering phase shifts.

The scattering phase shifts can be obtained by matching the logarithmic derivative for solutions inside and outside the muffin tin at the muffin-tin radius, $r=R_{\mathrm{MT}}$, as detailed in
Ref. [18]. The free scatterer DOS can also be easily computed from properties of the radial wave function. From a self-consistent DOS calculation, partitioned by both atom and angular momentum $l$, we therefore have all the components needed to approximate $\left\langle I^{2}\right\rangle$ for each atom type. These quantities can then be multiplied by $N\left(E_{F}\right)$ to give each $\eta_{j}$.

Gaspari-Gyorffy theory was implemented in the ELK code [19] in Ref. [20]; this modified version of the code is used in this work to calculate $\eta_{H}$ and $\eta_{X}$. The traditional way to use these approximate Hopfield parameters (or "Gaspari-Gyorffy electron-phonon coupling estimates") is to calculate the average phonon frequency and use this to estimate $\lambda$ via Eq. (1) [or, in our case, Eq. (2)]. This $\lambda$ value can in turn be used to obtain $T_{c}$ from the McMillan [13] or Allen-Dynes [21] equations. However, the phonon calculation required to obtain $\left\langle\omega^{2}\right\rangle$ means that this approach would be too expensive to be useful in a high-throughput screening scenario. Therefore, in this work, we do not obtain direct estimates of $\lambda$ or $T_{c}$ from Gaspari-Gyorffy theory and instead use the $\eta_{j}$ values themselves as descriptors in our $T_{c}$ model.

\section{Electron-phonon coupling calculations}

At several stages during this work electron-phonon coupling calculations are performed using density functional perturbation theory (DFPT) in the QUANTUM ESPRESSO code $[22,23]$ and the results treated using Migdal-Eliashberg theory [24] to obtain $T_{c}$ values from first principles. Within the theory, an electron in an occupied state $|n, \mathbf{k}\rangle$ is coupled to an unoccupied state $|m, \mathbf{k}+\mathbf{q}\rangle$ by a phonon with momentum $\mathbf{q}$ and frequency $\omega_{\mathbf{q}, v}$. At the temperatures of interest for superconductivity, we will have $\omega_{\mathbf{q} v} \sim k_{b} T \ll 1 \mathrm{eV}$, so the initial and final electronic states will lie very close to the Fermi energy. However, on the finite k-point grids used in DFT calculations, Kohn-Sham states will rarely lie this close to the Fermi level. To overcome this, we follow the method used in Ref. [25] and smear out the electronic energies using Gaussians with a characteristic smearing width $\sigma$, chosen according to the procedure outlined in Ref. [26].

Both the initial $(\mathbf{k})$ and final $(\mathbf{k}+\mathbf{q})$ states must be present in the k-point grid. To ensure that this is the case, we take our k-point grids to be fixed multiples of the q-point grids, increasing the multiplicative factor until convergence is achieved (typically this results in k-point grids larger than $30 \times 30 \times 30$ ). Coupling strengths are then interpolated onto a finer q-point grid (eight times larger in each direction) to construct the Eliashberg function, $\alpha^{2} F(\omega)$. From $\alpha^{2} F(\omega)$, we obtain the superconducting critical temperature either by direct solution of the Eliashberg equations using the ELK code [19] or using the Allen-Dynes equation [21]. We compare these two methods in Sec. IV F.

Throughout this work, we profiled the electron-phonon coupling calculations in the QUANTUM ESPRESSO code and implemented optimizations [27]. In particular, through optimized symmetrization of the electron-phonon matrix elements and evaluation of Gaussian smearing, we reduced the time to calculate $T_{c}$ for our $F m \overline{3} m-\mathrm{LaH}_{10}$ test system by a factor of 8 . Combined with the selective ability of our screening method, this near-order-of-magnitude speedup enables us to perform the present wide-ranging study. 


\section{III. $T_{c}$ MODEL AND TRAINING PHASE}

Our $T_{c}$ model is a Gaussian process regression model, initially trained using a set of 160 structures and corresponding $T_{c}$ values from the literature (collected from Refs. [20,26,2871]). The model inputs are the Gaspari-Gyorffy electronphonon coupling estimates for hydrogen and element $X\left(\eta_{H}\right.$ and $\left.\eta_{X}\right)$, the mass of atom $X$ in atomic units $\left(M_{X}\right)$, the total DOS at the Fermi energy $\left[N\left(E_{F}\right)\right.$, normalized by cell volume], and the hydrogen DOS divided by the total DOS at the Fermi energy $\left[N_{H}\left(E_{F}\right) / N\left(E_{F}\right)\right]$. Throughout this work, whether the structure originated from the literature or from our own searches, these inputs were computed using the modified version of ELK [19] from Ref. [20]. In cases where a literature structure was not given at the same pressure as $T_{c}$ was reported, the structure was relaxed at the correct pressure using CASTEP [10]. References [1-3,72] and the data tables within were found to be helpful for identifying additional points to include in the original literature set.

The model was trained via optimization of model parameters and hyperparameters in MATLAB [73] and was tested using nested cross-validation, with an inner loop used to optimize the parameters and an outer loop used to monitor the fit of the resulting model to unseen data. The correlation was evaluated in this way, repeated over several different random splittings of the data each time.

The overall process used in the training phase was iterative; structures at a given pressure were selected for further study based on the predictions of the model, with $T_{c}$ values for the best predicted structures calculated explicitly using DFPT and fed back into the model's training set for use in the next iteration (provided the structure was found to be dynamically stable). The model was then retrained and predictions were made for a set of search structures at the next pressure until all pressures had been considered. To make performing a large number of electron-phonon calculations feasible, a relatively sparse q-point sampling was used in the training stage, chosen to reproduce the known result for $F m \overline{3} m-\mathrm{LaH}_{10}[26,74]$. This corresponds to a q-point spacing of $2 \pi \times 0.15 \AA^{-1}$ (a $2 \times 2 \times 2$ grid for $F m \overline{3} m-\mathrm{LaH}_{10}$ at $200 \mathrm{GPa}$ ). In keeping with the initial literature training set, the Allen-Dynes equation was used to estimate $T_{c}$ throughout the training phase.

Our method is summarized in Fig. 1. Figure 2 shows the number of structures considered in total at each stage of the training process-given the relatively large cost of electronphonon calculations (even in high-throughput operation), this figure highlights the importance of the stability filtering and model-based screening steps in our workflow. In total, 119 new DFPT data points were added to the training set in this work. Predictions for all previously considered search structures were recalculated using the final model to ensure nothing of interest had been missed in earlier iterations. Most of the electron-phonon calculations performed in the training phase were for structures with a high predicted $T_{c}$ according to our model; however, occasionally these calculations were performed for structures with midrange or low $T_{c}$ predictions in order to improve the behavior of the model. The correlation of the predicted $T_{c}$ values with the calculated $T_{c}$ values across the training set decreased slightly on addition of more data (see Fig. 1). This is not surprising for two reasons: (1) as

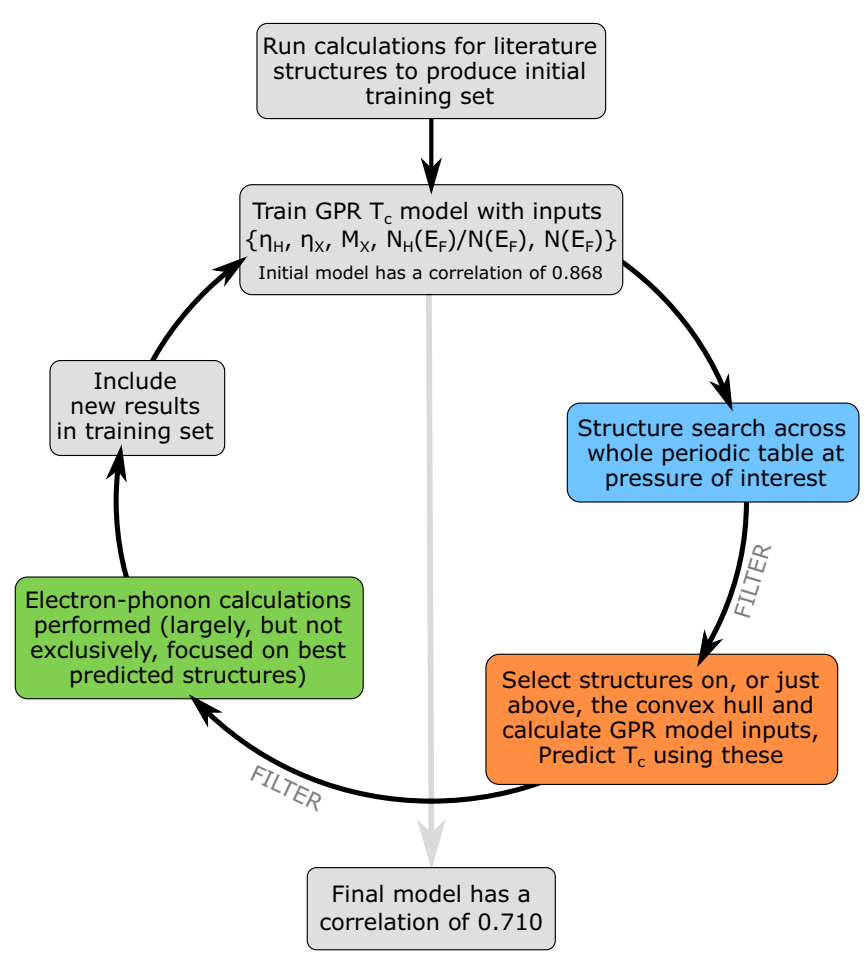

FIG. 1. A flowchart summarizing our methodology.

mentioned previously, the results we add to the training set are computed in a high-throughput manner and therefore will be somewhat underconverged compared to typical values found in the literature, and (2) the final training set contains a wider range of elements and pressures than the original literaturebased training set.

During the training of our model, we were able to efficiently rediscover a number of binary hydrides (with relatively high $T_{c}$ values) which had been reported previously, including the structures $\operatorname{Im} \overline{3} m-\mathrm{H}_{3} \mathrm{~S}[44,45,75]$,

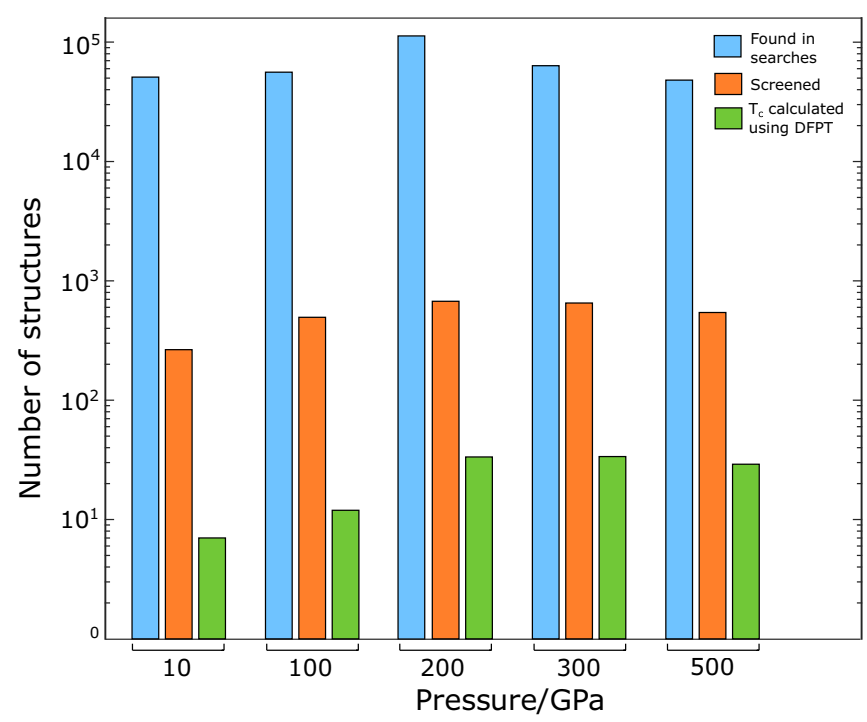

FIG. 2. A summary of the number of structures studied at each stage of the training process-note the logarithmic scale. 
$\operatorname{Im} \overline{3} m-\mathrm{LaH}_{6} \quad$ [76], I4/mmm- $\mathrm{AcH}_{12} \quad$ [28], $\quad$ Im $\overline{3} m-\mathrm{SeH}_{3}$ [46], $R \overline{3} m-\mathrm{SrH}_{6}$ [72], $R \overline{3} m-\mathrm{LiH}_{6} \quad[77,78], \quad F m \overline{3} m-\mathrm{LaH}_{10}$ $[26,50,74,76,79,80], \quad F m \overline{3} m-\mathrm{YH}_{10} \quad[26,76], \quad \operatorname{Im} \overline{3} m-\mathrm{ScH}_{6}$ $[57,76,81], \quad P 6_{3} / m m c-\mathrm{ThH}_{9} \quad[63], \quad R \overline{3} m-\mathrm{SrH}_{10} \quad$ [82], $P m \overline{3} m-\mathrm{SiH}_{3}$ [58], $C 2 / m-\mathrm{LaH}_{7}$ [50], Im $\overline{3} m-\mathrm{CaH}_{6}$ [35], $\operatorname{Im} \overline{3} m-\mathrm{MgH}_{6}$ [83], $F m \overline{3} m-\mathrm{ThH}_{10}$ [62,63], and the stoichiometries $\mathrm{KH}_{6}$ [49], $\mathrm{LaH}_{8}$ [50,79], $\mathrm{BaH}_{12}$ [70], $\mathrm{LaH}_{5}$ [50], $\mathrm{AcH}_{10}$ [28], $\mathrm{LiH}_{8}$ [77], $\mathrm{LaH}_{11}$ [50], $\mathrm{MgH}_{12}$ [84], $\mathrm{YH}_{9}$ [76,85], and $\mathrm{ScH}_{12}$ [57]. Although found previously, only half of these 26 stoichiometries appeared in our original training set; the other half were repredicted, highlighting the capabilities of our method. We also identified a number of other systems with the potential to exhibit high- $T_{c}$ superconductivity. The most promising systems overall are studied further in Sec. IV.

\section{FOCUSED SEARCHES AND FINAL RESULTS}

From the $T_{c}$ results obtained during training, the most promising candidate systems could be identified and studied in more depth. More focused structure searches were performed for hydrides of $\mathrm{Na}, \mathrm{Ca}, \mathrm{La}, \mathrm{Ac}$, and $\mathrm{K}$ at $100 \mathrm{GPa}$, hydrides of $\mathrm{La}, \mathrm{Ac}, \mathrm{S}, \mathrm{Mg}$, and $\mathrm{Na}$ at $200 \mathrm{GPa}$, hydrides of $\mathrm{Li}, \mathrm{Sr}, \mathrm{K}, \mathrm{Mg}, \mathrm{Na}$, and $\mathrm{Sc}$ at $300 \mathrm{GPa}$, and hydrides of $\mathrm{Li}, \mathrm{Sr}$, $\mathrm{Mg}, \mathrm{Na}, \mathrm{Yb}, \mathrm{Y}$, and $\mathrm{Ca}$ at $500 \mathrm{GPa}$. For these calculations, the earlier symmetry constraints were relaxed, but all other parameters remained the same. These composition choices reflect a bias towards elements from the left-hand side of the periodic table amongst the most promising candidates from the training stage. However, this is not to say that only the compositions that we study further in this work are capable of supporting high- $T_{c}$ superconductivity. Future studies, with a different focus, could potentially identify more candidates. No additional searches were performed at $10 \mathrm{GPa}$ as the highest $T_{c}$ calculated at this pressure in the training phase was only $12-15 \mathrm{~K}$ (belonging to $F m \overline{3} m-\mathrm{CaH}_{2}$ ) and a large proportion of the 245 structures screened were only weakly metallic (in fact, 48 of them were insulating).

On completion of the focused searches, we again calculated $\eta_{H}, \eta_{X}, N\left(E_{F}\right)$, and $N_{H}\left(E_{F}\right) / N\left(E_{F}\right)$ for the stable and metastable structures found. Stoichiometries with $E_{\text {stoic }} \leqslant 25$ $\mathrm{meV} /$ f.u. were selected for further study. For the stoichiometries on the hull $\left(E_{\text {stoic }}=0\right)$, two to five of the most stable structures were chosen. For the selected off-hull stoichiometries, only the lowest energy structure $\left(E_{\text {struc }}=0\right)$ was chosen. The inputs were then fed into the final $T_{c}$ model trained in Sec. III and fully converged electron-phonon calculations were performed for the structures with the highest predicted $T_{c}$ values at each pressure (as well as for the most promising candidates already identified during training). These results are shown in Figs. 3 and 4. Promising structures that remained dynamically stable after convergence of the q-point grids are listed in Table I. We find near room-temperature superconductors at every pressure considered, which we elaborate on in the following sections. Unless otherwise stated, all critical temperatures reported in the following sections are from direct solution of the Eliashberg equations.

\section{A. $100 \mathrm{GPa}$}

Of particular note at $100 \mathrm{GPa}$ is a $P m \overline{3} m$ structure of $\mathrm{NaH}_{6}$, a stoichiometry which we find to lie above the convex

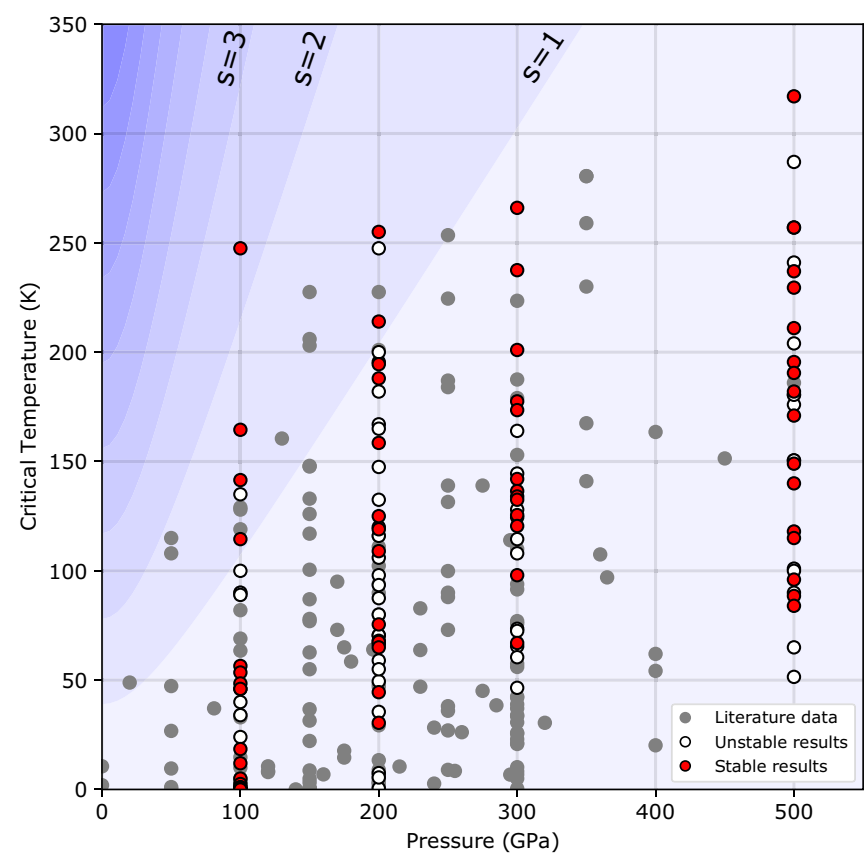

FIG. 3. Critical temperatures obtained from converged DFPT calculations for the most promising candidates according to our screening process (calculated using the Allen-Dynes equation to facilitate comparison with the literature). Both dynamically stable and dynamically unstable results are shown. The background is shaded according to the figure of merit $S$, introduced in Ref. [6].

hull, in agreement with Ref. [92] (although they find that the $P m \overline{3} m$ structure is not the lowest energy structure until $150 \mathrm{GPa}$ ). This structure consists of a cubic lattice of $\mathrm{H}$ octahedra with $\mathrm{Na}$ at the body-centered positions (see Fig. 5). Indeed, as we will see throughout this work, many of the best candidate systems contain hydrogen in such polyhedral clusters. While experimental synthesis of sodium polyhydrides has been demonstrated [93], superconductivity in the system seems understudied given its promise here with a calculated $T_{c}$ of $248-279 \mathrm{~K}$. This places the structure at a crucial position in pressure- $T_{c}$ space, strongly influencing the apparent low-pressure trend of maximum $T_{c}$ (see Figs. 3 and 4) and hinting at the exciting possibility of other low-pressure high- $T_{c}$ superconductors. Such high-temperature superconductivity is only possible with both high average phonon frequencies and strong electron-phonon coupling. Indeed, the logarithmic average phonon frequency, $\omega_{\mathrm{ln}}=870 \mathrm{~cm}^{-1}$, and electron-phonon coupling constant, $\lambda=2.54$, of $P m \overline{3} m-\mathrm{NaH}_{6}$ at $100 \mathrm{GPa}$ are comparable to the values for the $F m \overline{3} m-\mathrm{LaH}_{10}$ clathrate superconductor at $250 \mathrm{GPa}\left(\omega_{\mathrm{ln}}=871 \mathrm{~cm}^{-1}, \lambda=\right.$ 2.29 [50]). The ability of $P m \overline{3} m-\mathrm{NaH}_{6}$ to maintain a high average phonon frequency at such low pressures is due to its compact structure, with a shortest hydrogen-hydrogen bond length of only $d_{\mathrm{HH}}=0.85 \AA$ (shown in Fig. 5) - significantly shorter than in $F m \overline{3} m$ - $\mathrm{LaH}_{10}$, where $d_{\mathrm{HH}}>1 \AA$ up to pressures as high as $300 \mathrm{GPa}$ [94].

Also of interest at $100 \mathrm{GPa}$ is a cagelike $\operatorname{Im} \overline{3} m$ structure of $\mathrm{CaH}_{6}$ (see Fig. 3 of Ref. [35]). As was the case with $\mathrm{NaH}_{6}$, this stoichiometry is found to be slightly above the static-lattice convex hull, in agreement with previous calculations [35]. 
TABLE I. Allen-Dynes and Eliashberg $T_{c}$ values for dynamically stable superconductors found in this work, along with calculated $\lambda$ and stability measures. Structures that are underlined have a calculated $T_{c}$ above $100 \mathrm{~K}$ and have either not been reported in the literature before or have not had their superconducting properties studied before (to the best of our knowledge); more details are given in the Supplemental Material [90]. Structures are available online [91].

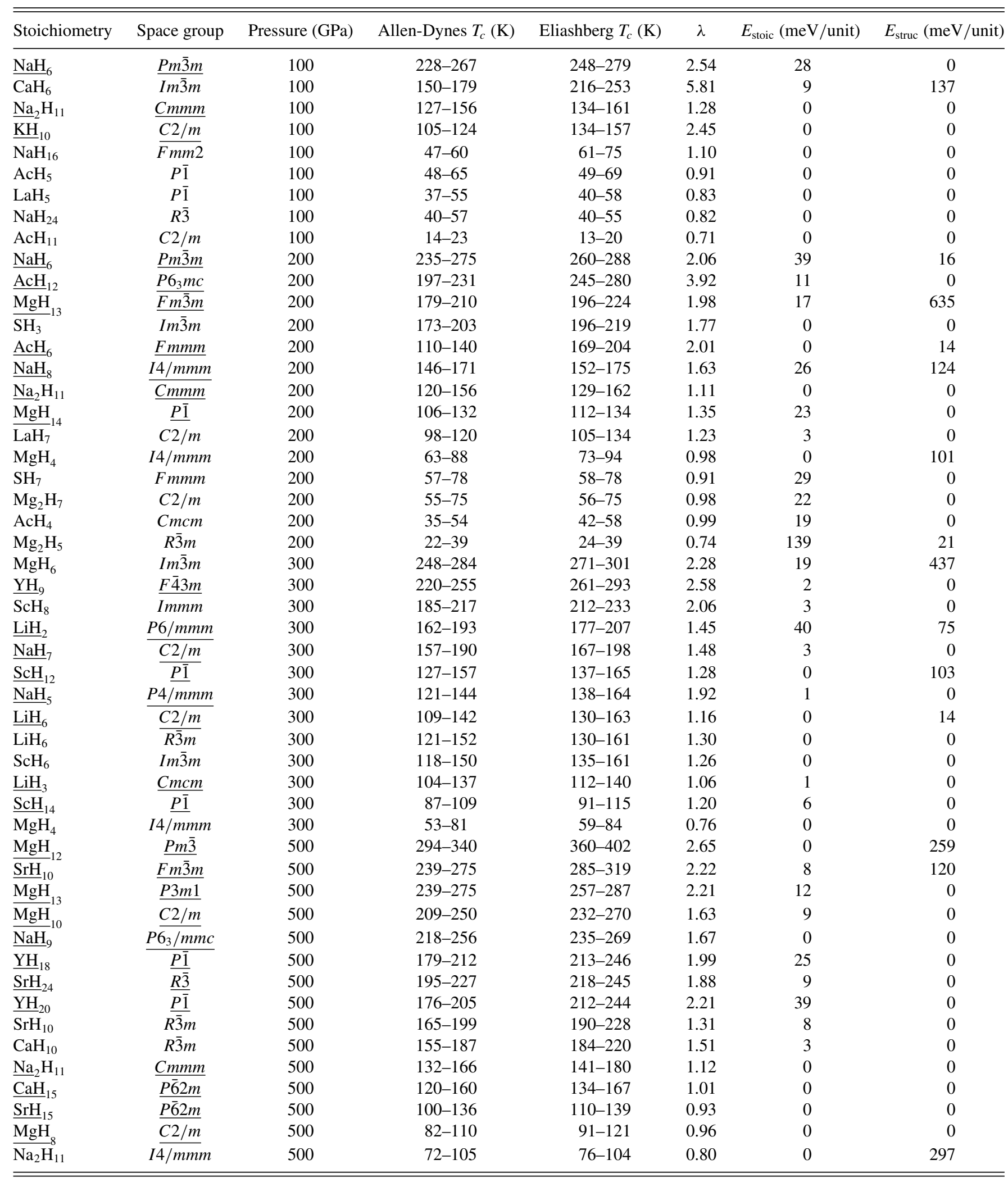




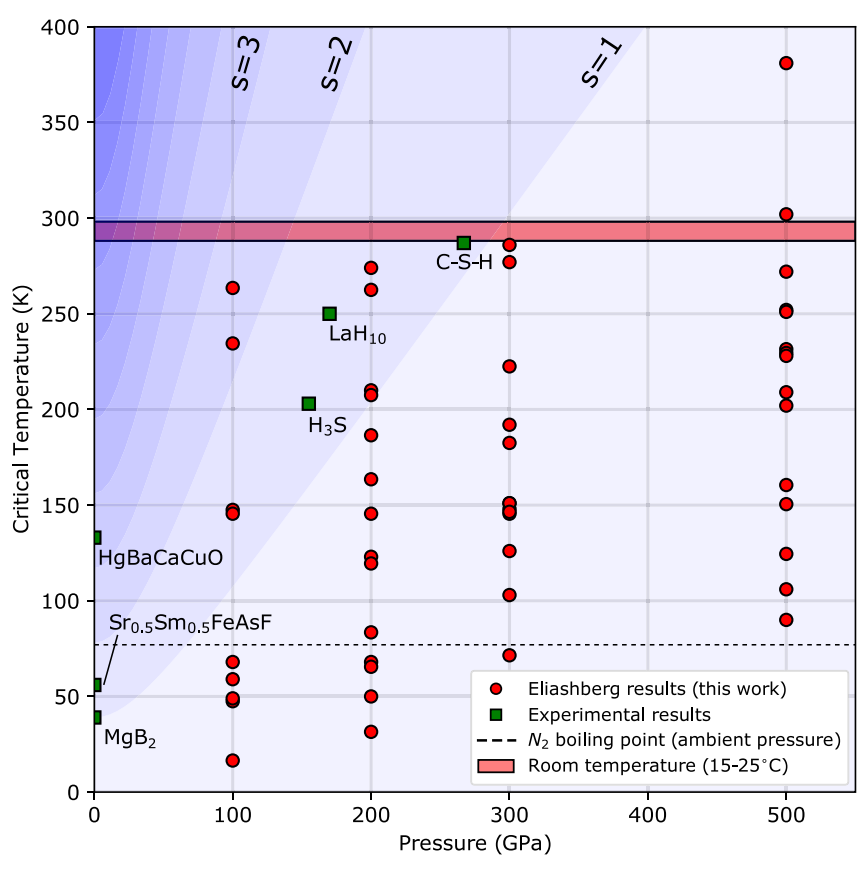

FIG. 4. As Fig. 3, but showing the Eliashberg results from Table I (dynamically stable structures only) alongside experimental results for specific superconductors (in order of increasing $T_{c}$ : [86-88,74,75,89].

However, despite strong electron-phonon coupling, its critical temperature $(216-253 \mathrm{~K})$ is found to be slightly lower than that of $P m \overline{3} m-\mathrm{NaH}_{6}$ due to a lower average phonon frequency. Indeed, our $\mathrm{CaH}_{6}$ phonons are significantly softer than those calculated at $150 \mathrm{GPa}$ in Ref. [35], resulting in a much higher $\lambda$ value (5.81 vs 2.69 from [35]), suggesting that the structure is on the verge of a mechanical instability at $100 \mathrm{GPa}$. Figure 6 illustrates this, highlighting the strong dependence of both $\lambda$ and the Allen-Dynes $T_{c}$ on the low-frequency portion of $\alpha^{2} F(\omega)$ and, in particular, the appearance of a peak in $\alpha^{2} F(\omega)$ at very low frequencies. This is unsurprising as the AllenDynes equation is known to be sensitive to small changes in $\alpha^{2} F(\omega)$ at low frequencies [in particular, the functional derivative $\delta T_{c}(\mathrm{AD}) / \delta \alpha^{2} F(\omega)$ diverges as $\omega \rightarrow 0$ [95], in this case towards $-\infty]$. However, the full Eliashberg calculation of $T_{c}$ is much less sensitive and the $100 \mathrm{GPa}$ result calculated here $(216-253 \mathrm{~K})$ is comparable to the critical temperature

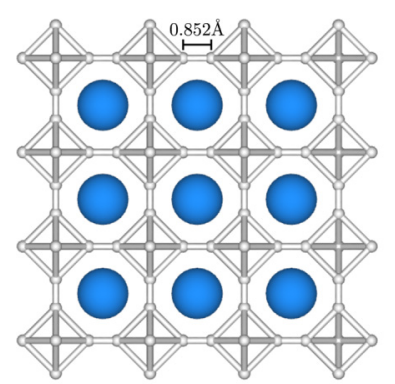

(a)

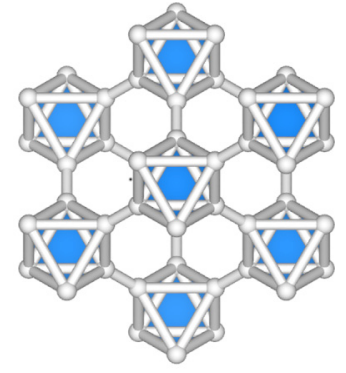

(b)
FIG. 5. The $100 \mathrm{GPa}$ structure of $P m \overline{3} m \mathrm{NaH}_{6}$ as viewed along the (a) [100] and (b) [111] directions of the standardized cell.

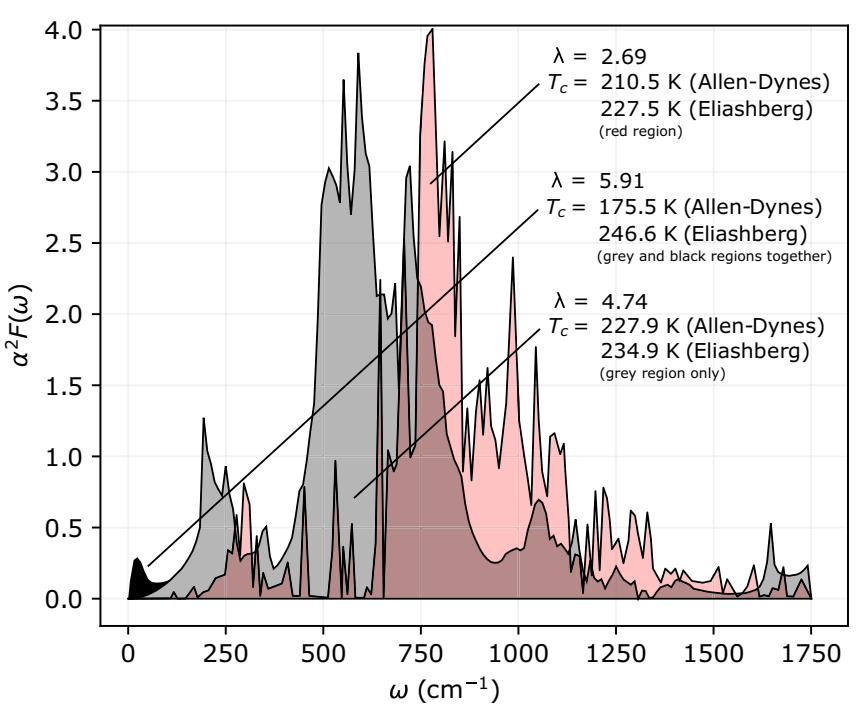

FIG. 6. Gray and black: The Eliashberg function of $\operatorname{Im} \overline{3} m \mathrm{CaH}_{6}$ at $100 \mathrm{GPa}$, demonstrating the suppression of the Allen-Dynes $T_{c}$ relative to the one obtained by solving the Eliashberg equations. This suppression is due to electrons coupling to soft phonon modes. Red: The Eliashberg function of the same material at $150 \mathrm{GPa}$ from Ref. [35].

of 220-235 K calculated at $150 \mathrm{GPa}$ in Ref. [35], also via solution of the Eliashberg equations.

Figure 7 also demonstrates that while the next best structures, $C m m m-\mathrm{Na}_{2} \mathrm{H}_{11}$ and $C 2 / m-\mathrm{KH}_{10}$, have similar average phonon frequencies to $P m \overline{3} m-\mathrm{NaH}_{6}$, they do not exhibit such high coupling strengths. This leads to lower $T_{c}$ values of 134 $161 \mathrm{~K}$ and $134-157 \mathrm{~K}$, respectively. $\mathrm{KH}_{10}$ adopts a cagelike structure, while the $\mathrm{Na}_{2} \mathrm{H}_{11}$ structure contains broken octahedral hydrogen clusters (see Fig. 7 inset). Superconductivity in

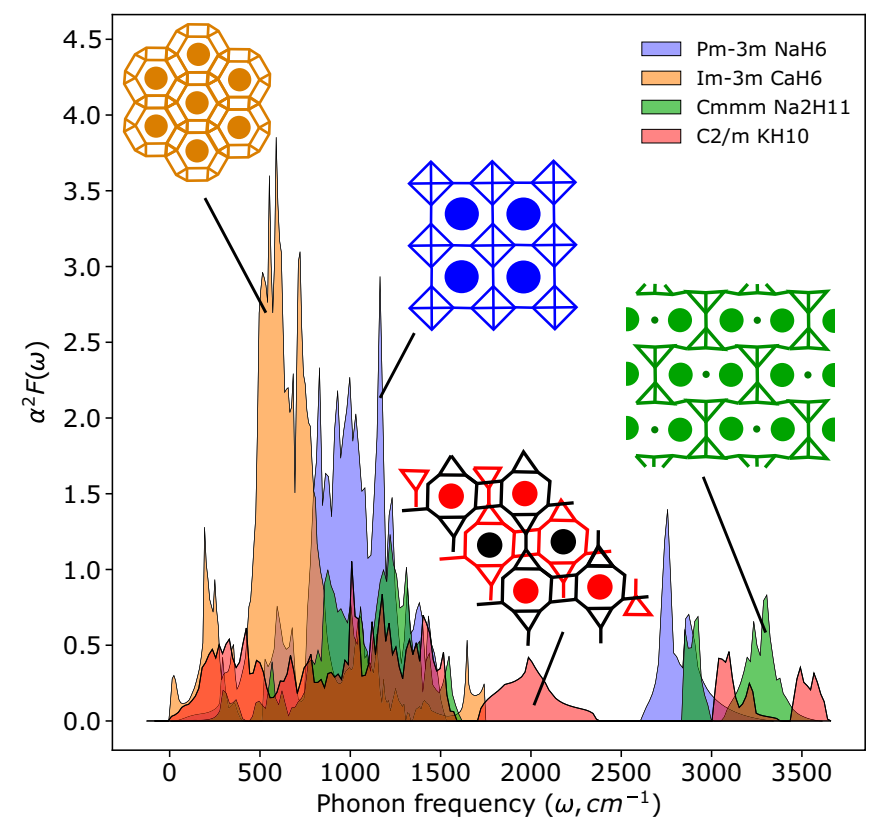

FIG. 7. The highest- $T_{c}$ structures and corresponding Eliashberg functions at $100 \mathrm{GPa}$. 


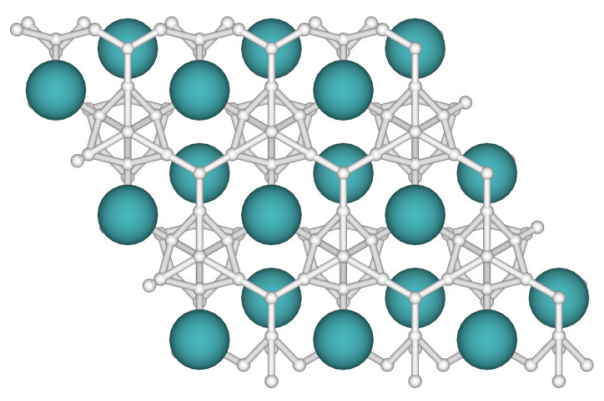

FIG. 8. The $200 \mathrm{GPa} P 6_{3} m c$ structure of $\mathrm{AcH}_{12}$ as viewed along the [001] direction of the standardized cell.

the $\mathrm{KH}_{10}$ stoichiometry has been studied previously [70]; it was found to be on the convex hull at $150 \mathrm{GPa}$ (and off-hull at $50 \mathrm{GPa}$ ) where an Allen-Dynes $T_{c}$ of $148 \mathrm{~K}$ was calculated for an Immm structure. Reference [96] found $\mathrm{KH}_{10}$ to be above the convex hull at $100 \mathrm{GPa}$ and instead found metastable metallic structures of other stoichiometries, but superconductivity was not directly investigated.

\section{B. 200 GPa}

At $200 \mathrm{GPa}$, the $P m \overline{3} m$ structure of $\mathrm{NaH}_{6}$ remains the highest $T_{c}$ structure found, rising slightly from its $100 \mathrm{GPa}$ value to 260-288 K. However, as pressure increases we find this stoichiometry to be less stable with respect to decomposition. Similarly to Ref. [28], we find several actinium hydrides to be high-temperature superconductors at this pressure, most notably a $P 6_{3} m c$ structure of $\mathrm{AcH}_{12}$ with a critical temperature of $245-280 \mathrm{~K}$. This structure is best described as cagelike, but with hydrogen atoms concentrated along specific channels along the $c$ axis (see Fig. 8).

$200 \mathrm{GPa}$ also marks the appearance of magnesium hydrides, which become increasingly prevalent in our results with pressure. Of particular note is a cubic structure of $\mathrm{MgH}_{13}$ with the space group $F m \overline{3} m$, which possesses a slightly higher calculated $T_{c}(196-224 \mathrm{~K})$ than the experimentally verified [75] $I m \overline{3} m-\mathrm{H}_{3} \mathrm{~S}$ at the same pressure $(196-219 \mathrm{~K})$. The $\mathrm{MgH}_{13}$ structure consists of axis-aligned cuboctahedral hydrogen clusters (see Fig. 9). In agreement with Ref. [84], we find $\mathrm{MgH}_{13}$ to lie above the convex hull at $200 \mathrm{GPa}$.

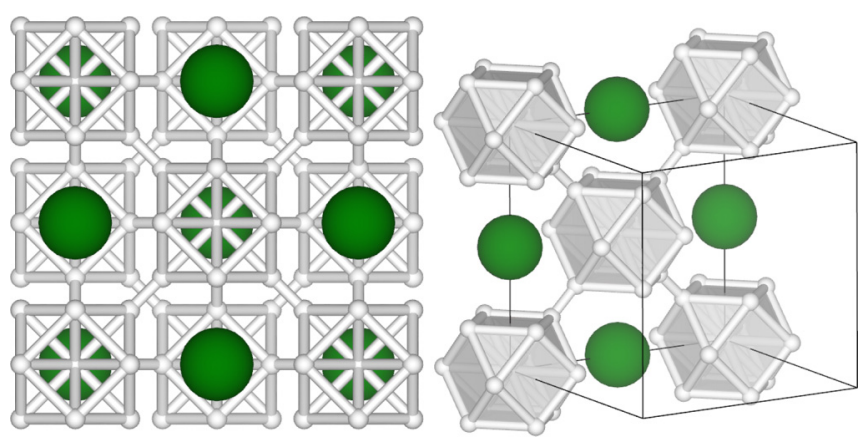

FIG. 9. The $200 \mathrm{GPa} F m \overline{3} m$ structure of $\mathrm{MgH}_{13}$. The structure consists of $\mathrm{Mg}$ atoms and axis-aligned cuboctahedra of hydrogen in a checkerboard pattern. Each cuboctahedra has an additional hydrogen atom at its center to make up the necessary 13 .

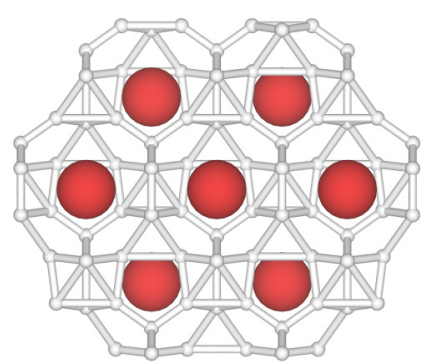

(a)

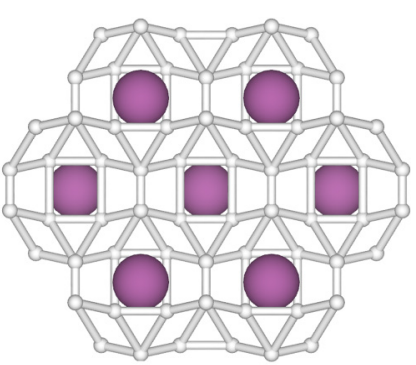

(b)
FIG. 10. (a) The $300 \mathrm{GPa} F \overline{4} 3 m$ structure of $\mathrm{YH}_{9}$ as viewed along the [110] direction of the standardized cell. (b) The $300 \mathrm{GPa}$ Immm structure of $\mathrm{ScH}_{8}$ as viewed along the [100] direction of the standardized cell.

They report significantly lower critical temperatures for onhull structures.

A notable absence from the $200 \mathrm{GPa}$ results is $\mathrm{LaH}_{10}$. Several structures of $\mathrm{LaH}_{10}$ (including the experimentally verified $F m \overline{3} m$ structure [74,80]) were found in our structure searches and flagged as good candidates by our $T_{c}$ model, but were dynamically unstable at the harmonic level. This has been noted previously for the cubic structure [26,97,98]. We elaborate on our treatment of structures with dynamical instabilities in Sec. IV E.

\section{C. $300 \mathrm{GPa}$}

At $300 \mathrm{GPa}$, an $\operatorname{Im} \overline{3} m$ structure of $\mathrm{MgH}_{6}$ exhibits the highest $T_{c}$ value, 271-301 $\mathrm{K}$ (in agreement with previous work [83]). This structure can be obtained by substituting $\mathrm{Ca}$ for $\mathrm{Mg}$ in the $\mathrm{CaH}_{6}$ structure investigated at $100 \mathrm{GPa}$. By instead substituting only half of the $\mathrm{Ca}$ atoms at $200 \mathrm{GPa}$, it has been reported that one obtains an even higher critical temperature ternary superconductor [99]. Hybridizing compatible binary crystal structures in this way could provide an efficient method to design future high-temperature ternary hydride superconductors.

With a critical temperature of 261-293 K, a cagelike $F \overline{4} 3 m$ structure of $\mathrm{YH}_{9}$ [shown in Fig. 10(a)] is the next highest temperature superconductor found at $300 \mathrm{GPa}$. This stoichiometry (although with $P 6_{3} / m m c$ symmetry) has been synthesized experimentally, confirming theoretical predictions [76], and was found to exhibit a critical temperature of $243 \mathrm{~K}$ at $201 \mathrm{GPa}$ [85]. Y-H systems have been extensively studied [26,100102] with critical temperatures in excess of $200 \mathrm{~K}$ predicted over large pressure ranges.

We find that an Immm structure of $\mathrm{ScH}_{8}$ with a similar motif to $F \overline{4} 3 m-\mathrm{YH}_{9}$ [see Fig. 10 (b)] is also a high-temperature superconductor at this pressure with a $T_{c}$ of 212-233 K. This is significantly higher than the (Allen-Dynes) value of $\sim 115 \mathrm{~K}$ obtained in Ref. [103]; we elaborate on the source of this discrepancy in the Supplemental Material [90].

The next structure of note is a metastable $P 6 / \mathrm{mmm}$ structure of $\mathrm{LiH}_{2}$, which is interesting both because of its relatively low hydrogen content and because its structure is analogous to the well-known ambient-pressure superconductor, $\mathrm{MgB}_{2}$ [86] (see Fig. 11). Superconductivity in Li-H systems has 


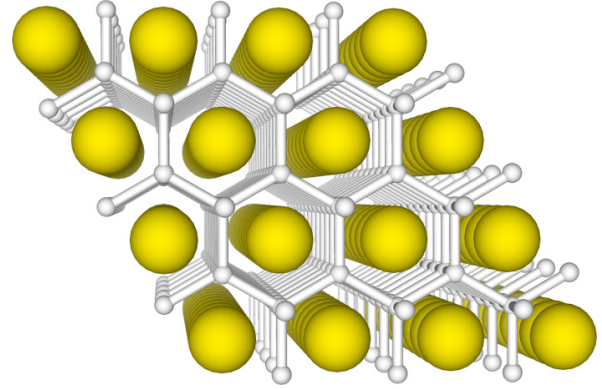

FIG. 11. The $300 \mathrm{GPa} P 6 / \mathrm{mmm}$ structure of $\mathrm{LiH}_{2}$ as viewed with perspective along the [001] direction of the standardized cell.

been investigated previously at lower pressures [77], where it was found that the $\mathrm{LiH}_{2}$ stoichiometry did not exhibit superconductivity at $150 \mathrm{GPa}$. We find that the $\mathrm{LiH}_{2}$ stoichiometry lies above the static-lattice convex hull at $300 \mathrm{GPa}$. However, we find this stoichiometry is stabilized (and lies on the hull, in agreement with Ref. [104]) when using harder pseudopotentials with denser k-point grids.

\section{D. $500 \mathrm{GPa}$}

As we increase pressure further, hydrides with higher hydrogen content can be metallized. In particular, at $500 \mathrm{GPa}$ we see the appearance of several $\mathrm{MgH}_{n}$ structures with $n>10$. The highest critical temperature of these belongs to $\mathrm{MgH}_{12}$, which we find to be on the convex hull at this pressure, where a $P m \overline{3}$ structure [see Fig. 12(a)] exhibits hot superconductivity with a critical temperature of 360-402 K $\left(87-129^{\circ} \mathrm{C}\right)$. This extremely high critical temperature is due to strong electron-phonon coupling that persists over the entire phonon spectrum, extending to very high frequencies (see Fig. 13). Despite increased hydrogen content, $P 3 m 1-\mathrm{MgH}_{13}$ [see Fig. 12(b)] exhibits a lower $T_{c}$ of $257-287 \mathrm{~K}$ due to reduced (but still extended) electron-phonon coupling (see Fig. 13). Superconductivity in the $\mathrm{Mg}-\mathrm{H}$ system appears to be enhanced substantially with increased pressure; the $\mathrm{MgH}_{12}$ stoichiometry has been previously investigated at lower pressures [84], where it was also found to lie on the convex hull, but with a much reduced $T_{c}$ of $47-60 \mathrm{~K}$ at $140 \mathrm{GPa}$.

At $500 \mathrm{GPa}$, we also find that a high-symmetry metastable $F m \overline{3} m$ phase of $\mathrm{SrH}_{10}$ exhibits room-temperature superconductivity with a $T_{c}$ of $285-319 \mathrm{~K}\left(12-46^{\circ} \mathrm{C}\right)$. This is

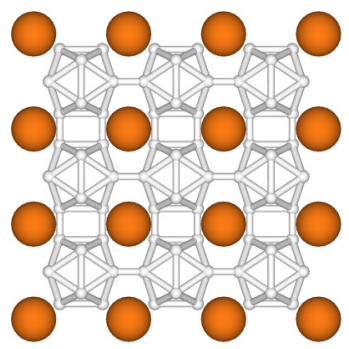

(a)

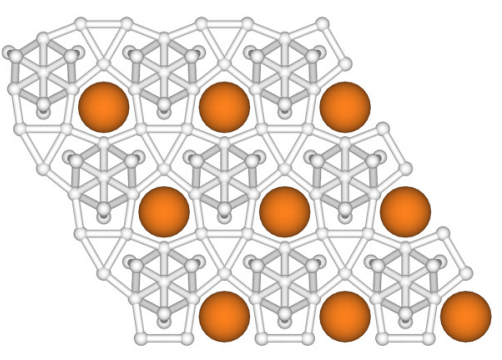

(b)
FIG. 12. (a) The $500 \mathrm{GPa} P m \overline{3}$ structure of $\mathrm{MgH}_{12}$ as viewed along the [010] direction of the standardized cell. (b) The $500 \mathrm{GPa}$ $P 3 m 1$ structure of $\mathrm{MgH}_{13}$ as viewed along the [001] direction of the standardized cell.

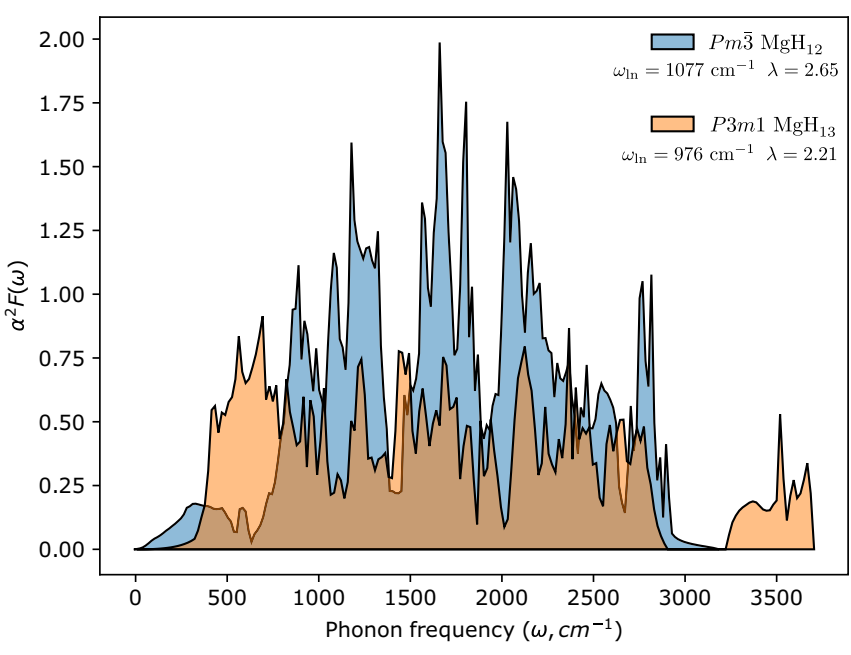

FIG. 13. The Eliashberg functions of the $P m \overline{3}$ structure of $\mathrm{MgH}_{12}$ (a hot superconductor with a $T_{c}$ of $360-402 \mathrm{~K}$ ) and the $P 3 m 1$ structure of $\mathrm{MgH}_{13}$, both at $500 \mathrm{GPa}$.

significantly higher than the 190-228 K we calculate for the ground-state structure of this stoichiometry, which we find to have $R \overline{3} m$ symmetry in agreement with previous calculations at $300 \mathrm{GPa}$ [105]. A $T_{c}$ of $259 \mathrm{~K}$ had been calculated for the $R \overline{3} m$ structure previously at this lower pressure [82].

\section{E. Dynamically unstable structures}

Structures that are found to be dynamically unstable correspond to saddle points, rather than minima, of the potential energy surface. Such structures can be stabilized by anharmonic or thermal effects, as is the case in $F m \overline{3} m-\mathrm{LaH}_{10}$ at $200 \mathrm{GPa}[26,74,106]$, and locating them is the goal of sp-AIRSS [12]. The calculations required to filter out, or correctly treat [107-110], dynamically unstable superconductors are expensive; the solution of this problem will be important in the further development of high-throughput workflows. However, one can roughly establish the promise of a saddle-point superconductor by simply neglecting unstable modes in the calculation of the Eliashberg function [26,111]. We provide the results of this procedure for dynamically unstable structures that were flagged by the final model in the Supplemental Materil [90] (see also Fig. 3), along with a short discussion of the validity of this procedure.

\section{F. A modified Allen-Dynes equation}

Having a large number of superconductors for which the Eliashberg equations have been solved directly (see Table I) provides a unique opportunity to test the Allen-Dynes equation. This comparison is made in Fig. 14(a), where it is clear to see that, while the Allen-Dynes result correlates well with the Eliashberg result, it systematically underestimates its value (at least for the binary hydride superconductors studied in this work). We fit a modified version of the Allen-Dynes equation of the form

$$
T_{c}=T_{c}^{(\mathrm{AD})}(a+b \lambda)
$$

to the data given in Table I, which gives $a=1.0061$ and $b=0.0663$. As we can see in Fig. 14(b) this removes the 

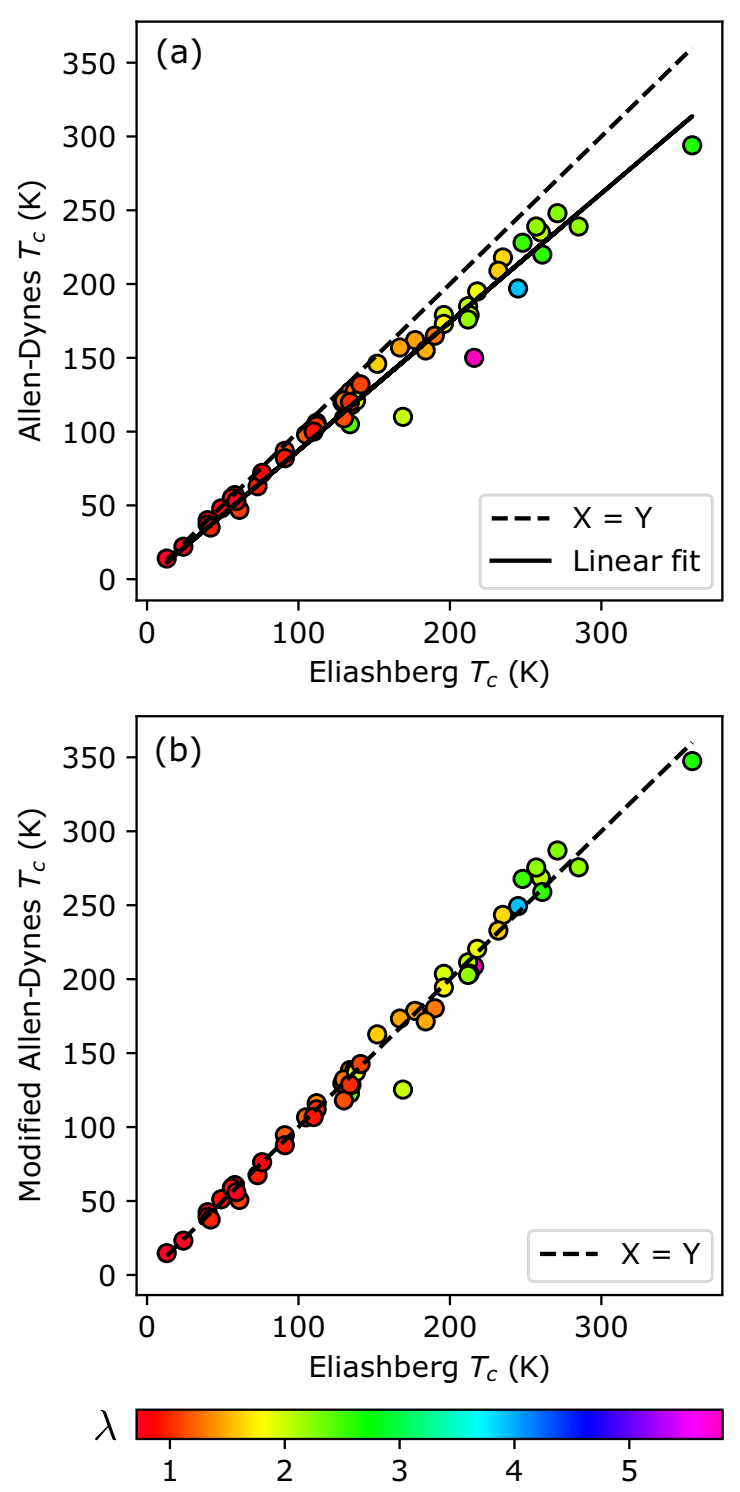

FIG. 14. (a) Allen-Dynes critical temperatures, plotted against critical temperatures from solution of the Eliashberg equations (data from Table I). (b) The same as (a), but using the modified AllenDynes equation [Eq. (4)].

systematic underestimation and slightly reduces the variance of the prediction. However, given access to $\alpha^{2} F(\omega)$, we recommend that the Eliashberg equations be solved directly, to remove the need for approximate $T_{c}$ predictors entirely.

\section{CONCLUSIONS}

In this work, we demonstrate a high-throughput method to efficiently discover high- $T_{c}$ binary hydrides. We construct a $T_{c}$ model based on physically motivated descriptors, trained initially on superconductivity data from the literature and iteratively updated using the results of our own DFPT calculations. Following extensive structure searching, a two-step screening process (based on stability criteria and the predictions of our model) allows us to identify energetically competitive high- $T_{c}$ candidates from the large volume of search data. The best candidates include hydrides of sodium, calcium, actinium, lanthanum, magnesium, yttrium, scandium, lithium, and strontium.

We have performed a total of $240 T_{c}$ calculations using DFPT, split roughly equally between the training phase and final results. This was made possible by optimizing the QUANTUM ESPRESSO electron-phonon code. In the final results, we identify 36 dynamically stable superconductors with $T_{c}>100 \mathrm{~K}$ of which 18 have $T_{c}>200 \mathrm{~K}$ (see Table I). To the best of our knowledge, superconductivity has not been investigated previously in 27 of the 36 (see Table I and the Supplemental Material [90]). These findings add considerably to the known pressure- $T_{c}$ behavior of the binary hydrides. Of particular note, we find a $P m \overline{3} m$ structure of $\mathrm{NaH}_{6}$ to have a $T_{c}$ of $248-279 \mathrm{~K}$ at $100 \mathrm{GPa}$, suggesting the exciting possibility of other low-pressure high- $T_{c}$ hydride superconductors. We also identify $P m \overline{3}-\mathrm{MgH}_{12}$ and $F m \overline{3} m-\mathrm{SrH}_{10}$ as above-roomtemperature superconductors at $500 \mathrm{GPa}$, as well as several near-room-temperature superconductors at lower pressures.

Throughout this work, our aim has been to consider as wide a range of binary compositions as possible; since our focus is on breadth, we make no claim that this study is exhaustive. Despite this, we identify a large number of high- $T_{c}$ candidates, suggesting the binaries have more to offer in future, more focused, studies. We also note that the highest critical temperature results at each pressure arise from metastable structures or off-hull stoichiometries. Many of these were introduced into the study during the training phase by focusing on high-symmetry structures using sp-AIRSS. This suggests that the additional freedom afforded by allowing some degree of metastability can reveal higher critical temperature superconductors. For example, in the case of $\mathrm{SrH}_{10}$ at $500 \mathrm{GPa}$ we see that the ground state $R \overline{3} \mathrm{~m}$ structure has a critical temperature nearly $100 \mathrm{~K}$ lower than a metastable $F m \overline{3} m$ structure. Exploring avenues such as metastability will be important in future work in order to push the boundaries of high-temperature superconductivity.

Future work on superconducting hydrides is likely to focus on ternary hydrides and higher order systems. Given the increased complexity of these systems, high-throughput screening approaches, such as the one presented here, are likely to become increasingly important. Our high-throughput methodology could be extended to ternary hydrides, although it may be desirable to redefine these systems as effective binaries in order make use of the extensive binary hydride literature data during model training.

\section{ACKNOWLEDGMENTS}

A.M.S. is funded by an EPSRC studentship. M.J.H. acknowledges the EPSRC Centre for Doctoral Training in Computational Methods for Materials Science for funding under Grant No. EP/L015552/1. R.J.N. is supported by EPSRC under Critical Mass Grant No. EP/P034616/1 and the UKCP consortium Grant No. EP/P022596/1. C.J.P. was supported by the Royal Society through a Royal Society Wolfson Research Merit award. This work has been performed using resources provided by the Cambridge Tier- 2 system operated by the University of Cambridge Research Computing Service funded by EPSRC Tier-2 capital Grant No. EP/P020259/1. 
[1] D. Duan, Y. Liu, Y. Ma, Z. Shao, B. Liu, and T. Cui, Structure and superconductivity of hydrides at high pressures, Natl. Sci. Rev. 4, 121 (2017).

[2] E. Zurek and T. Bi, High-temperature superconductivity in alkaline and rare earth polyhydrides at high pressure: A theoretical perspective, J. Chem. Phys. 150, 050901 (2019).

[3] J. A. Flores-Livas, L. Boeri, A. Sanna, G. Profeta, R. Arita, and M. Eremets, A perspective on conventional high-temperature superconductors at high pressure: Methods and materials, Phys. Rep. 856, 1 (2020).

[4] L. Boeri and G. B. Bachelet, Viewpoint: The road to room-temperature conventional superconductivity, J. Phys.: Condens. Matter 31, 234002 (2019).

[5] A. R. Oganov, C. J. Pickard, Q. Zhu, and R. J. Needs, Structure prediction drives materials discovery, Nat. Rev. Mater. 4, 331 (2019).

[6] C. J. Pickard, I. Errea, and M. I. Eremets, Superconducting hydrides under pressure, Annu. Rev. Condens. Matter Phys. 11, 57 (2020).

[7] G. D. Gaspari and B. L. Gyorffy, Electron-Phonon Interactions, $d$ Resonances, and Superconductivity in Transition Metals, Phys. Rev. Lett. 28, 801 (1972).

[8] C. J. Pickard and R. J. Needs, High-Pressure Phases of Silane, Phys. Rev. Lett. 97, 045504 (2006).

[9] C. J. Pickard and R. J. Needs, $A b$ initio random structure searching, J. Phys.: Condens. Matter 23, 053201 (2011).

[10] S. J. Clark, M. D. Segall, C. J. Pickard, P. J. Hasnip, M. I. J. Probert, K. Refson, and M. C. Payne, First principles methods using CASTEP, Z. Kristallogr.-Cryst. Mater. 220, 567 (2005).

[11] J. P. Perdew, K. Burke, and M. Ernzerhof, Generalized Gradient Approximation Made Simple, Phys. Rev. Lett. 77, 3865 (1996).

[12] B. Monserrat, N. D. Drummond, P. Dalladay-Simpson, R. T. Howie, P. López Ríos, E. Gregoryanz, C. J. Pickard, and R. J. Needs, Structure and Metallicity of Phase V of Hydrogen, Phys. Rev. Lett. 120, 255701 (2018).

[13] W. L. McMillan, Transition temperature of strong-coupled superconductors, Phys. Rev. 167, 331 (1968).

[14] J. J. Hopfield, Angular momentum and transition-metal superconductivity, Phys. Rev. 186, 443 (1969).

[15] J. C. Slater, Wave functions in a periodic potential, Phys. Rev. 51, 846 (1937).

[16] D. A. Papaconstantopoulos, B. Klein, M. J. Mehl, and W. E. Pickett, Cubic $\mathrm{H}_{3} \mathrm{~S}$ around $200 \mathrm{GPa}$ : An atomic hydrogen superconductor stabilized by sulfur, Phys. Rev. B 91, 184511 (2015)

[17] P.-H. Chang, S. Silayi, D. Papaconstantopoulos, and M. Mehl, Pressure-induced high-temperature superconductivity in hypothetical $\mathrm{H}_{3} \mathrm{X}(\mathrm{X}=\mathrm{As}, \mathrm{Se}, \mathrm{Br}, \mathrm{Sb}, \mathrm{Te}$, and $\mathrm{I})$ in the $\mathrm{H}_{3} \mathrm{~S}$ structure with $\operatorname{Im} \overline{3} m$ symmetry, J. Phys. Chem. Solids 139, 109315 (2020).

[18] J. J. Sakurai and J. Napolitano, Scattering theory, in Modern Quantum Mechanics, 2nd ed. (Cambridge University Press, Cambridge, UK, 2017), pp. 386-445.

[19] The ELK FP-LAPW code, http://elk.sourceforge.net/.

[20] M. J. Hutcheon, A. M. Shipley, and R. J. Needs, Predicting novel superconducting hydrides using machine learning approaches, Phys. Rev. B 101, 144505 (2020).
[21] P. B. Allen and R. C. Dynes, Transition temperature of strongcoupled superconductors reanalyzed, Phys. Rev. B 12, 905 (1975).

[22] P. Giannozzi, S. Baroni, N. Bonini, M. Calandra, R. Car, C. Cavazzoni, D. Ceresoli, G. L. Chiarotti, M. Cococcioni, I. Dabo et al., QUANTUM ESPRESSO: A modular and opensource software project for quantum simulations of materials, J. Phys.: Condens. Matter 21, 395502 (2009).

[23] P. Giannozzi, O. Andreussi, T. Brumme, O. Bunau, M. B. Nardelli, M. Calandra, R. Car, C. Cavazzoni, D. Ceresoli, M. Cococcioni et al., Advanced capabilities for materials modelling with QUANTUMESPRESSO, J. Phys.: Condens. Matter 29, 465901 (2017).

[24] G. M. Eliashberg, Interactions between electrons and lattice vibrations in a superconductor, Sov. Phys. JETP 11, 696 (1960).

[25] M. Wierzbowska, S. de Gironcoli, and P. Giannozzi, Origins of low-and high-pressure discontinuities of $T_{c}$ in niobium, arXiv:cond-mat/0504077.

[26] A. M. Shipley, M. J. Hutcheon, M. S. Johnson, R. J. Needs, and C. J. Pickard, Stability and superconductivity of lanthanum and yttrium decahydrides, Phys. Rev. B 101, 224511 (2020).

[27] The optimizations to QUANTUM ESPRESSO resulting from this work have been submitted to the developers (see https://gitlab. $\mathrm{com} / \mathrm{miicck} / \mathrm{q}$-e for implementation details).

[28] D. V. Semenok, A. G. Kvashnin, I. A. Kruglov, and A. R. Oganov, Actinium hydrides $\mathrm{AcH}_{10}, \mathrm{AcH}_{12}$, and $\mathrm{AcH}_{16}$ as high-temperature conventional superconductors, J. Phys. Chem. Lett. 9, 1920 (2018).

[29] P. Hou, X. Zhao, F. Tian, D. Li, D. Duan, Z. Zhao, B. Chu, B. Liu, and T. Cui, High pressure structures and superconductivity of $\mathrm{AlH}_{3}\left(\mathrm{H}_{2}\right)$ predicted by first principles, RSC Adv. 5, 5096 (2015)

[30] Y. Fu, X. Du, L. Zhang, F. Peng, M. Zhang, C. J. Pickard, R. J. Needs, D. J. Singh, W. Zheng, and Y. Ma, High-pressure phase stability and superconductivity of pnictogen hydrides and chemical trends for compressed hydrides, Chem. Mater. 28, 1746 (2016).

[31] K. Abe and N. W. Ashcroft, Crystalline diborane at high pressures, Phys. Rev. B 84, 104118 (2011).

[32] S. Yu, Q. Zeng, A. R. Oganov, C. Hu, G. Frapper, and L. Zhang, Exploration of stable compounds, crystal structures, and superconductivity in the Be-H system, AIP Adv. 4, 107118 (2014).

[33] C.-H. Hu, A. R. Oganov, Q. Zhu, G.-R. Qian, G. Frapper, A. O. Lyakhov, and H.-Y. Zhou, Pressure-Induced Stabilization and Insulator-Superconductor Transition of BH, Phys. Rev. Lett. 110, 165504 (2013).

[34] Y. Ma, D. Duan, D. Li, Y. Liu, F. Tian, H. Yu, C. Xu, Z. Shao, B. Liu, and T. Cui, High-pressure structures and superconductivity of bismuth hydrides, arXiv:1511.05291.

[35] H. Wang, S. T. John, K. Tanaka, T. Iitaka, and Y. Ma, Superconductive sodalite-like clathrate calcium hydride at high pressures, Proc. Natl. Acad. Sci. USA 109, 6463 (2012).

[36] N. P. Salke, M. M. D. Esfahani, Y. Zhang, I. A. Kruglov, J. Zhou, Y. Wang, E. Greenberg, V. B. Prakapenka, J. Liu, A. R. Oganov, and J.-F. Lin, Synthesis of clathrate cerium superhydride $\mathrm{CeH}_{9}$ at 80-100 GPa with atomic hydrogen sublattice, Nat. Commun. 10, 1 (2019). 
[37] S. Yu, X. Jia, G. Frapper, D. Li, A. R. Oganov, Q. Zeng, and L. Zhang, Pressure-driven formation and stabilization of superconductive chromium hydrides, Sci. Rep. 5, 17764 (2015).

[38] C. Heil, G. B. Bachelet, and L. Boeri, Absence of superconductivity in iron polyhydrides at high pressures, Phys. Rev. B 97, 214510 (2018).

[39] M. M. Davari Esfahani, A. R. Oganov, H. Niu, and J. Zhang, Superconductivity and unexpected chemistry of germanium hydrides under pressure, Phys. Rev. B 95, 134506 (2017).

[40] G. Gao, A. R. Oganov, A. Bergara, M. Martinez-Canales, T. Cui, T. litaka, Y. Ma, and G. Zou, Superconducting High Pressure Phase of Germane, Phys. Rev. Lett. 101, 107002 (2008).

[41] G. Zhong, C. Zhang, X. Chen, Y. Li, R. Zhang, and H. Lin, Structural, electronic, dynamical, and superconducting properties in dense $\mathrm{GeH}_{4}\left(\mathrm{H}_{2}\right)_{2}$, J. Phys. Chem. C 116, 5225 (2012).

[42] D. Duan, F. Tian, Y. Liu, X. Huang, D. Li, H. Yu, Y. Ma, B. Liu, and T. Cui, Enhancement of $T_{c}$ in the atomic phase of iodine-doped hydrogen at high pressures, Phys. Chem. Chem. Phys. 17, 32335 (2015).

[43] A. Shamp and E. Zurek, Superconducting high-pressure phases composed of hydrogen and iodine, J. Phys. Chem. Lett. 6, 4067 (2015).

[44] D. Duan, Y. Liu, F. Tian, D. Li, X. Huang, Z. Zhao, H. Yu, B. Liu, W. Tian, and T. Cui, Pressure-induced metallization of dense $\left(\mathrm{H}_{2} \mathrm{~s}\right)_{2} \mathrm{H}_{2}$ with high- $T_{c}$ superconductivity, Sci. Rep. 4, 6968 (2014).

[45] I. Errea, M. Calandra, C. J. Pickard, J. Nelson, R. J. Needs, Y. Li, H. Liu, Y. Zhang, Y. Ma, and F. Mauri, High-Pressure Hydrogen Sulfide from First Principles: A Strongly Anharmonic Phonon-Mediated Superconductor, Phys. Rev. Lett. 114, 157004 (2015).

[46] S. Zhang, Y. Wang, J. Zhang, H. Liu, X. Zhong, H.-F. Song, G. Yang, L. Zhang, and Y. Ma, Phase diagram and high-temperature superconductivity of compressed selenium hydrides, Sci. Rep. 5, 15433 (2015).

[47] Y. Liu, D. Duan, F. Tian, C. Wang, G. Wu, Y. Ma, H. Yu, D. Li, B. Liu, and T. Cui, Prediction of stoichiometric $\mathrm{PoH}_{n}$ compounds: Crystal structures and properties, RSC Adv. 5, 103445 (2015).

[48] X. Zhong, H. Wang, J. Zhang, H. Liu, S. Zhang, H.-F. Song, G. Yang, L. Zhang, and Y. Ma, Tellurium Hydrides at High Pressures: High-Temperature Superconductors, Phys. Rev. Lett. 116, 057002 (2016).

[49] D. Zhou, X. Jin, X. Meng, G. Bao, Y. Ma, B. Liu, and T. Cui, $A b$ initio study revealing a layered structure in hydrogen-rich $\mathrm{KH}_{6}$ under high pressure, Phys. Rev. B 86, 014118 (2012).

[50] I. A. Kruglov, D. V. Semenok, H. Song, R. Szczęśniak, I. A. Wrona, R. Akashi, M. M. Davari Esfahani, D. Duan, T. Cui, A. G. Kvashnin, and A. R. Oganov, Superconductivity of $\mathrm{LaH}_{10}$ and $\mathrm{LaH}_{16}$ polyhydrides, Phys. Rev. B 101, 024508 (2020).

[51] G. Gao, R. Hoffmann, N. W. Ashcroft, H. Liu, A. Bergara, and Y. Ma, Theoretical study of the ground-state structures and properties of niobium hydrides under pressure, Phys. Rev. B 88, 184104 (2013).

[52] D. Zhou, D. V. Semenok, H. Xie, X. Huang, D. Duan, A. Aperis, P. M. Oppeneer, M. Galasso, A. I. Kartsev, A. G. Kvashnin, A. R. Oganov, and T. Cui, High-pressure synthesis of magnetic neodymium polyhydrides, J. Am. Chem. Soc. 142, 2803 (2020).
[53] Y. Liu, D. Duan, X. Huang, F. Tian, D. Li, X. Sha, C. Wang, H. Zhang, T. Yang, B. Liu, and T. Cui, Structures and properties of osmium hydrides under pressure from first principle calculation, J. Phys. Chem. C 119, 15905 (2015).

[54] D. Zhou, D. V. Semenok, D. Duan, H. Xie, W. Chen, X. Huang, X. Li, B. Liu, A. R. Oganov, and T. Cui, Superconducting praseodymium superhydrides, Sci. Adv. 6, eaax6849 (2020)

[55] Y. Liu, D. Duan, F. Tian, C. Wang, Y. Ma, D. Li, X. Huang, B. Liu, and T. Cui, Stability and properties of the $\mathrm{Ru}-\mathrm{H}$ system at high pressure, Phys. Chem. Chem. Phys. 18, 1516 (2016).

[56] Y. Ma, D. Duan, D. Li, Y. Liu, F. Tian, X. Huang, Z. Zhao, H. $\mathrm{Yu}, \mathrm{B}$. Liu, and T. Cui, The unexpected binding and superconductivity in $\mathrm{SbH}_{4}$ at high pressure, arXiv:1506.03889.

[57] X. Ye, N. Zarifi, E. Zurek, R. Hoffmann, and N. Ashcroft, High hydrides of scandium under pressure: Potential superconductors, J. Phys. Chem. C 122, 6298 (2018).

[58] X. Jin, X. Meng, Z. He, Y. Ma, B. Liu, T. Cui, G. Zou, and H.-k. Mao, Superconducting high-pressure phases of disilane, Proc. Natl. Acad. Sci. USA 107, 9969 (2010).

[59] M. M. D. Esfahani, Z. Wang, A. R. Oganov, H. Dong, Q. Zhu, S. Wang, M. S. Rakitin, and X.-F. Zhou, Superconductivity of novel tin hydrides $\left(\mathrm{Sn}_{n} \mathrm{H}_{m}\right)$ under pressure, Sci. Rep. 6, 22873 (2016)

[60] Q. Zhuang, X. Jin, T. Cui, Y. Ma, Q. Lv, Y. Li, H. Zhang, X. Meng, and K. Bao, Pressure-stabilized superconductive ionic tantalum hydrides, Inorg. Chem. 56, 3901 (2017).

[61] X. Li, H. Liu, and F. Peng, Crystal structures and superconductivity of technetium hydrides under pressure, Phys. Chem. Chem. Phys. 18, 28791 (2016).

[62] A. G. Kvashnin, D. V. Semenok, I. A. Kruglov, I. A. Wrona, and A. R. Oganov, High-temperature superconductivity in a Th-H system under pressure conditions, ACS Appl. Mater. Interfaces 10, 43809 (2018).

[63] D. V. Semenok, A. G. Kvashnin, A. G. Ivanova, V. Svitlyk, V. Y. Fominski, A. V. Sadakov, O. A. Sobolevskiy, V. M. Pudalov, I. A. Troyan, and A. R. Oganov, Superconductivity at $161 \mathrm{~K}$ in thorium hydride $\mathrm{ThH}_{10}$ : Synthesis and properties, Mater. Today 33, 36 (2020).

[64] I. A. Kruglov, A. G. Kvashnin, A. F. Goncharov, A. R. Oganov, S. S. Lobanov, N. Holtgrewe, S. Jiang, V. B. Prakapenka, E. Greenberg, and A. V. Yanilkin, Uranium polyhydrides at moderate pressures: Prediction, synthesis, and expected superconductivity, Sci. Adv. 4, eaat9776 (2018).

[65] X. Li and F. Peng, Superconductivity of pressure-stabilized vanadium hydrides, Inorg. Chem. 56, 13759 (2017).

[66] S. Zheng, S. Zhang, Y. Sun, J. Zhang, J. Lin, G. Yang, and A. Bergara, Structural and superconducting properties of tungsten hydrides under high pressure, Frontiers Phys. 6, 101 (2018).

[67] L.-L. Liu, H.-J. Sun, C. Wang, and W.-C. Lu, High-pressure structures of yttrium hydrides, J. Phys.: Condens. Matter 29, 325401 (2017).

[68] X.-F. Li, Z.-Y. Hu, and B. Huang, Phase diagram and superconductivity of compressed zirconium hydrides, Phys. Chem. Chem. Phys. 19, 3538 (2017).

[69] K. Abe, High-pressure properties of dense metallic zirconium hydrides studied by ab initio calculations, Phys. Rev. B 98, 134103 (2018).

[70] D. V. Semenok, I. A. Kruglov, I. A. Savkin, A. G. Kvashnin, and A. R. Oganov, On distribution of superconductivity in 
metal hydrides, Curr. Opin. Solid State Mater. Sci. 24, 100808 (2020).

[71] Q. Gu, P. Lu, K. Xia, J. Sun, and D. Xing, High-temperature superconducting phase of $\mathrm{HBr}$ under pressure predicted by first-principles calculations, Phys. Rev. B 96, 064517 (2017).

[72] T. Bi, N. Zarifi, T. Terpstra, and E. Zurek, The search for superconductivity in high pressure hydrides, Reference Module in Chemistry, Molecular Sciences and Chemical Engineering (Elsevier, New York, 2019).

[73] MATLAB GPR fitting documentation, https://uk. mathworks.com/help/stats/fitrgp.html.

[74] A. Drozdov, P. Kong, V. Minkov, S. Besedin, M. Kuzovnikov, S. Mozaffari, L. Balicas, F. Balakirev, D. Graf, V. Prakapenka et al., Superconductivity at $250 \mathrm{~K}$ in lanthanum hydride under high pressures, Nature (London) 569, 528 (2019).

[75] A. Drozdov, M. Eremets, I. Troyan, V. Ksenofontov, and S. Shylin, Conventional superconductivity at 203 kelvin at high pressures in the sulfur hydride system, Nature (London) 525, 73 (2015).

[76] F. Peng, Y. Sun, C. J. Pickard, R. J. Needs, Q. Wu, and Y. Ma, Hydrogen Clathrate Structures in Rare Earth Hydrides at High Pressures: Possible Route to Room-Temperature Superconductivity, Phys. Rev. Lett. 119, 107001 (2017).

[77] Y. Xie, Q. Li, A. Oganov, and H. Wang, Superconductivity of lithium-doped hydrogen under high pressure, Acta Crystallogr., Section. C: Struct. Chem. 70, 104 (2014).

[78] R. T. Howie, O. Narygina, C. L. Guillaume, S. Evans, and E. Gregoryanz, High-pressure synthesis of lithium hydride, Phys. Rev. B 86, 064108 (2012).

[79] H. Liu, I. I. Naumov, R. Hoffmann, N. Ashcroft, and R. J. Hemley, Potential high- $T_{c}$ superconducting lanthanum and yttrium hydrides at high pressure, Proc. Natl. Acad. Sci. USA 114, 6990 (2017).

[80] M. Somayazulu, M. Ahart, A. K. Mishra, Z. M. Geballe, M. Baldini, Y. Meng, V. V. Struzhkin, and R. J. Hemley, Evidence for Superconductivity Above $260 \mathrm{~K}$ in Lanthanum Superhydride at Megabar Pressures, Phys. Rev. Lett. 122, 027001 (2019).

[81] K. Abe, Hydrogen-rich scandium compounds at high pressures, Phys. Rev. B 96, 144108 (2017).

[82] K. Tanaka, J. S. Tse, and H. Liu, Electron-phonon coupling mechanisms for hydrogen-rich metals at high pressure, Phys. Rev. B 96, 100502(R) (2017).

[83] X. Feng, J. Zhang, G. Gao, H. Liu, and H. Wang, Compressed sodalite-like $\mathrm{MgH}_{6}$ as a potential high-temperature superconductor, RSC Adv. 5, 59292 (2015).

[84] D. C. Lonie, J. Hooper, B. Altintas, and E. Zurek, Metallization of magnesium polyhydrides under pressure, Phys. Rev. B 87, 054107 (2013).

[85] P. Kong, V. Minkov, M. Kuzovnikov, S. Besedin, A. Drozdov, S. Mozaffari, L. Balicas, F. Balakirev, V. Prakapenka, E. Greenberg et al., Superconductivity up to $243 \mathrm{~K}$ in yttrium hydrides under high pressure, arXiv:1909.10482.

[86] J. Nagamatsu, N. Nakagawa, T. Muranaka, Y. Zenitani, and J. Akimitsu, Superconductivity at $39 \mathrm{~K}$ in magnesium diboride, Nature (London) 410, 63 (2001).

[87] G. Wu, Y. L. Xie, H. Chen, M. Zhong, R. H. Liu, B. C. Shi, Q. J. Li, X. F. Wang, T. Wu, Y. J. Yan et al., Superconductivity at $56 \mathrm{~K}$ in samarium-doped SrFeAsF, J. Phys.: Condens. Matter 21, 142203 (2009).
[88] A. Schilling, M. Cantoni, J. D. Guo, and H. R. Ott, Superconductivity above $130 \mathrm{~K}$ in the $\mathrm{Hg}-\mathrm{Ba}-\mathrm{Ca}-\mathrm{Cu}-\mathrm{O}$ system, Nature (London) 363, 56 (1993).

[89] E. Snider, N. Dasenbrock-Gammon, R. McBride, M. Debessai, H. Vindana, K. Vencatasamy, K. V. Lawler, A. Salamat, and R. P. Dias, Room-temperature superconductivity in a carbonaceous sulfur hydride, Nature (London) 586, 373 (2020).

[90] See Supplemental Material at http://link.aps.org/ supplemental/10.1103/PhysRevB.104.054501, which includes Refs. [112-121]. This contains a discussion of computational parameters for $\mathrm{ScH}_{8}$ and results for dynamically unstable superconductors alongside a brief discussion. Tables listing previous investigations (where they exist) of structures from this work are also provided along with convex hulls for various systems at various pressures.

[91] Crystal structures for the superconductors in Table 1. are available in the Cambridge online data repository, https://doi.org/ 10.17863/CAM.72574.

[92] P. Baettig and E. Zurek, Pressure-Stabilized Sodium Polyhydrides: $\mathrm{NaH}_{n}(n>1)$, Phys. Rev. Lett. 106, 237002 (2011).

[93] V. V. Struzhkin, D. Y. Kim, E. Stavrou, T. Muramatsu, H.-k. Mao, C. J. Pickard, R. J. Needs, V. B. Prakapenka, and A. F. Goncharov, Synthesis of sodium polyhydrides at high pressures, Nat. Commun. 7, 12267 (2016).

[94] C. Wang, S. Yi, and J.-H. Cho, Pressure dependence of the superconducting transition temperature of compressed $\mathrm{LaH}_{10}$, Phys. Rev. B 100, 060502(R) (2019).

[95] G. Bergmann and D. Rainer, The sensitivity of the transition temperature to changes in $\alpha^{2} f(\omega)$, Z. Phys. 263, 59 (1973).

[96] J. Hooper and E. Zurek, High pressure potassium polyhydrides: A chemical perspective, J. Phys. Chem. C 116, 13322 (2012).

[97] Z. M. Geballe, H. Liu, A. K. Mishra, M. Ahart, M. Somayazulu, Y. Meng, M. Baldini, and R. J. Hemley, Synthesis and stability of lanthanum superhydrides, Angew. Chem., Int. Ed. 57, 688 (2018).

[98] H. Liu, I. I. Naumov, Z. M. Geballe, M. Somayazulu, J. S. Tse, and R. J. Hemley, Dynamics and superconductivity in compressed lanthanum superhydride, Phys. Rev. B 98, 100102(R) (2018).

[99] W. Sukmas, P. Tsuppayakorn-aek, U. Pinsook, and T. Bovornratanaraks, Near-room-temperature superconductivity of $\mathrm{Mg} / \mathrm{Ca}$ substituted metal hexahydride under pressure, J. Alloys Compd. 849, 156434 (2020).

[100] C. Heil, S. di Cataldo, G. B. Bachelet, and L. Boeri, Superconductivity in sodalite-like yttrium hydride clathrates, Phys. Rev. B 99, 220502(R) (2019).

[101] I. A. Troyan, D. V. Semenok, A. G. Kvashnin, A. V. Sadakov, O. A. Sobolevskiy, V. M. Pudalov, A. G. Ivanova, V. B. Prakapenka, E. Greenberg, A. G. Gavriliuk et al., Anomalous high-temperature superconductivity in $\mathrm{YH}_{6}$, Adv. Mater. 33, 2006832 (2021).

[102] Y. Li, J. Hao, H. Liu, S. T. John, Y. Wang, and Y. Ma, Pressurestabilized superconductive yttrium hydrides, Sci. Rep. 5, 9948 (2015).

[103] S. Qian, X. Sheng, X. Yan, Y. Chen, and B. Song, Theoretical study of stability and superconductivity of $\mathrm{ScH}_{n}(n=4-8)$ at high pressure, Phys. Rev. B 96, 094513 (2017). 
[104] E. Zurek, R. Hoffmann, N. W. Ashcroft, A. R. Oganov, and A. O. Lyakhov, A little bit of lithium does a lot for hydrogen, Proc. Natl. Acad. Sci. USA 106, 17640 (2009).

[105] Y. Wang, H. Wang, S. T. John, T. Iitaka, and Y. Ma, Structural morphologies of high-pressure polymorphs of strontium hydrides, Phys. Chem. Chem. Phys. 17, 19379 (2015).

[106] I. Errea, F. Belli, L. Monacelli, A. Sanna, T. Koretsune, T. Tadano, R. Bianco, M. Calandra, R. Arita, F. Mauri, and J. A. Flores-Livas, Quantum crystal structure in the $250-\mathrm{K}$ superconducting lanthanum hydride, Nature (London) 578, 66 (2020).

[107] I. Errea, M. Calandra, C. J. Pickard, J. R. Nelson, R. J. Needs, Y. Li, H. Liu, Y. Zhang, Y. Ma, and F. Mauri, Quantum hydrogen-bond symmetrization in the superconducting hydrogen sulfide system, Nature (London) 532, 81 (2016).

[108] I. Errea, M. Calandra, and F. Mauri, Anharmonic free energies and phonon dispersions from the stochastic self-consistent harmonic approximation: Application to platinum and palladium hydrides, Phys. Rev. B 89, 064302 (2014).

[109] J. C. K. Hui and P. B. Allen, Effect of lattice anharmonicity on superconductivity, J. Phys. F 4, L42 (1974).

[110] V. Meregalli and S. Y. Savrasov, Electron-phonon coupling and properties of doped $\mathrm{BaBiO}_{3}$, Phys. Rev. B 57, 14453 (1998).

[111] X. Wan, H.-C. Ding, S. Y. Savrasov, and C.-G. Duan, Electron-phonon superconductivity near charge-density-wave instability in $\mathrm{LaO}_{0.5} \mathrm{~F}_{0.5} \mathrm{BiS}_{2}$ : Density-functional calculations, Phys. Rev. B 87, 115124 (2013).

[112] H. Xie, D. Duan, Z. Shao, H. Song, Y. Wang, X. Xiao, D. Li, F. Tian, B. Liu, and T. Cui, High-temperature superconductivity in ternary clathrate $\mathrm{YCaH}_{12}$ under high pressures, J. Phys.: Condens. Matter 31, 245404 (2019).
[113] Y. Sun, J. Lv, Y. Xie, H. Liu, and Y. Ma, Route to a Superconducting Phase Above Room Temperature in Electron-Doped Hydride Compounds under High Pressure, Phys. Rev. Lett. 123, 097001 (2019).

[114] R. Szczesniak and A. Durajski, Superconductivity well above room temperature in compressed $\mathrm{MgH}_{6}$, Front. Phys. 11, 117406 (2016).

[115] Y. Chen, H. Y. Geng, X. Yan, Y. Sun, Q. Wu, and X. Chen, Prediction of stable ground-state lithium polyhydrides under high pressures, Inorg. Chem. 56, 3867 (2017).

[116] J. A. Camargo-Martínez, G. I. González-Pedreros, and F. Mesa, The higher superconducting transition temperature $T_{c}$ and the functional derivative of $T_{c}$ with $\alpha^{2} F(\omega)$ for electron-phonon superconductors, J. Phys.: Condens. Matter 32, 505901 (2020).

[117] Y.-K. Wei, J.-N. Yuan, F. I. Khan, G.-F. Ji, Z.-W. Gu, and D.-Q. Wei, Pressure induced superconductivity and electronic structure properties of scandium hydrides using first principles calculations, RSC Adv. 6, 81534 (2016).

[118] J. S. Smith, S. Desgreniers, D. D. Klug, and S. T. John, Highdensity strontium hydride: An experimental and theoretical study, Solid State Commun. 149, 830 (2009).

[119] Z. Shao, D. Duan, Y. Ma, H. Yu, H. Song, H. Xie, D. Li, F. Tian, B. Liu, and T. Cui, Unique phase diagram and superconductivity of calcium hydrides at high pressures, Inorg. Chem. 58, 2558 (2019).

[120] H. Xie, Y. Yao, X. Feng, D. Duan, H. Song, Z. Zhang, S. Jiang, S. A. T. Redfern, V. Z. Kresin, C. J. Pickard, and T. Cui, Hydrogen Pentagraphenelike Structure Stabilized by Hafnium: A High-Temperature Conventional Superconductor, Phys. Rev. Lett. 125, 217001 (2020).

[121] J. Hooper, T. Terpstra, A. Shamp, and E. Zurek, Composition and constitution of compressed strontium polyhydrides, J. Phys. Chem. C 118, 6433 (2014). 